\title{
OXYGEN SUPPLY-A COST-COMPARISON STUDY
}

\author{
By JoHN S. HERON, B.A., M.D., F.A.C.A. ${ }^{*}$
}

THE demand for administration of oxygen for therapeutic purposes has progressively increased,-so that to-day it is seven times greater than it was ten years ago.

As anaesthetists, we use oxygen every day, but how many of us are aware of the cost of this agent that we employ so casually? At the Toronto East General and Orthopaedic Hospital we obtain oxygen in bulk From the storage units it is piped to the various hospital departments.

The theoretical advantages of an oxygen piping system from the standpoint of equpment and oxygen economies and better service to patients are well known.

I was currous to determine in dollars and cents how an oxygen piping system had affected this hospital. Therefore, I made a cost comparison of piped oxygen versus cylinder oxygen (by cylinder oxygen I refer to the $244 \mathrm{cu}$. $\mathrm{ft}$. size cylinder).

The cost of pipe-line installation has been estimated as follows:

1) New construction-such as in our new west wing: $\$ 50 .-\$ 60$. per outlet.

2) Old construction-such as in our old north wing:

a) exposed piping. $\$ 80 .-\$ 95$. per outlet

b) concealed piping. $\$ 95 .-\$ 125$. per outlet.

Our present monthly consumption of oxygen averages $80,000 \mathrm{cu}$. $\mathrm{ft}$. This costs 77 cents per $100 \mathrm{cu}$. ft.

The monthly bill for oxygen at the Toronto East General and Orthopaedic Hospital $=\$ 616.00$.

This volume of oxygen is comparable to that held in 328 cylinders of $244 \mathrm{cu}$. $\mathrm{ft}$. capacity. On a cylinder basis (excluding handling costs) $100 \mathrm{cu}$. ft. of oxygen costs 95 cents. The monthly bill for oxygen if obtained in cylinders would be $\$ 76000$ This represents a saving on oxygen used of $(760-616)=\$ 144.00$ per month,-based on the assumption that each cylinder is completely emptied,whereas in actual practice anywhere from 3 to $15 \%$ of the cylinder contents is returned in the "empty tank" which means that the saving is greater than represented here.

A cost study performed at this hospital revealed that the handling of one $244 \mathrm{cu}$. ft. cylinder of oxygen cost $\$ 1.25$. Therefore the cost of handling such cylinders for one month would be $328 \times 1.25=\$ 410.00$.

Consequently the cost of oxygen and handling it in cylinders $=$ $(760+410)=\$ 1,170.00$. Thus the oxygen piping system effects a monthly saving of $\$ 554.00$.

It has been estimated that the savings effected on labour alone will pay for

*Toronto East General and Orthopaedic Hospital 
the pipe line at the Toronto East General and Orthopaedic Hospital over a period of five years.

\section{Oxygen Tent}

To maintain a $40 \%$ concentration of oxygen in a tent $t$ 1e flow rate must be 12 litres/minute. At this rate three tanks are used in a 24 hcur period. The handling charges on the oxygen tanks required to maintain one tent in use for one week $=(1.25 \times 3 \times 7)=\$ 26.25$. The handling charges over two weeks will say for one oxygen outlet in a piped oxygen system.

\section{-Vursery}

One incubator in the nursery requires one tank of oxygen daily. The monthly saving effected by the use of piped oxygen in the nursery is $\$ 50.00$ per incubator - wh is obtained as follows:

1) \$37.50-handling charges for cylinders.

2) \$13.00-actual cost of oxygen consumed.

\section{Regulators}

When using large cylinders of oxygen it is necessary to employ two stage regulators-these cost $\$ 65.00$ each and may be overhauled for $\$ 25.00$.

The pressure in an oxygen piping system is only $50 \mathrm{lb}$. per square inch compared with $2000 \mathrm{lb}$. per square inch in a large oxygen cylinder. Therefore a relatively simple and inexpensive flowmeter may be used on the wall outlets of a central oxygen system. The cost of the flowrneters used at the Toronto East General and Orthopaedic Hospital is $\$ 16.55$ and these may be overhauled for $\$ 8.00$.

\section{Conclusion}

1) The provision of a continuous and adequate supply of oxygen has been effected at considerable saving to the hospital.

2) At the Toronto East General and Orthopaedic Hospital it is felt that in the modern hospital of fifty beds or more, a piped oxygen supply is as essential and as practical as a piped water supply. 